\title{
Effectiveness of systematic alphanumeric coded endoscopy for diagnosis of gastric intraepithelial neoplasia in a low socioeconomic population
}

Authors

Institutions
Nancy Roxana Machaca Quea', Fabian Emura' ${ }^{2,3,4}$, Fernando Barreda Bolaños ${ }^{5}$, Yuliana Salvador Arias', Fernando Antonio Arévalo Suárez ${ }^{6,7}$, Alejandro Piscoya Rivera ${ }^{7,8}$

Institutions are listed at the end of article. submitted 28. January 2016 accepted after revision 29. July 2016

\section{Bibliography}

Dol http://dx.doi.org/ 10.1055/s-0042-115408 Endoscopy International Open 2016; 04: E1083-E1089 (c) Georg Thieme Verlag KG Stuttgart · New York E-ISSN 2196-9736

Corresponding author Fabian Emura MD, PhD, FASGE Calle 134 No. 7-83 Office 341

Bogotá DC, Colombia Phone: +57-6271493 fabian@emuracenter.org
Background and study aims: In the Western world, gastric cancer (GC) usually presents at an advanced stage, carrying a high mortality rate. Studies have reported that $14 \%$ to $26 \%$ of GCs are missed at endoscopy up to 3 years before diagnosis. Systematic Alphanumeric Coded Endoscopy (SACE) has been proposed to improve quality of esophagogastroduodenoscopy (EGD) by facilitating a complete examination of the upper gastrointestinal tract. This prospective cross-sectional study was designed to determine the frequency of gastric intraepithelial neoplasia (GIN) by using the SACE approach in cohort of patients from low socioeconomic level. It also used non-targeted biopsies to evaluate the frequency of premalignant conditions.

Patients and methods: A total of 601 consecutive asymptomatic or dyspeptic patients were enrolled between January 2013 and November 2014 at the Huacho regional hospital in Peru. The SACE method proposed by Emura et al, which divides the stomach into 5 regions and 21 areas, was rou-

\section{Introduction}

$\nabla$

Gastric cancer (GC) is currently the fifth most common cancer in incidence and the third most common in mortality worldwide [1]. In the Western world, GC usually presents at an advanced stage, carrying a high mortality rate $[1,2]$. In Peru, as in other Latin American countries, GC has the highest incidence among all cancers with 16.8 cases per 100,000 inhabitants, and constitutes the main cause of cancer-related deaths $[2,3]$. Noteworthy, GC mostly affects people from low socioeconomic levels [4], with nearly $90 \%$ of cases diagnosed in an advanced stage when opportunities for curative therapy are limited [3].

Although esophagogastroduodenoscopy (EGD) can detect early-stage lesions, in practice, finding an early gastric cancer (EGC) is challenging, even for experts. In fact, studies have reported that $14 \%$ tinely used for diagnosis. Biopsy samples were obtained from any endoscopically detected focal lesion. To evaluate gastric premalignant conditions, 4 non-targeted biopsies were taken.

Results: A total of 573 patients were analyzed. The mean age was 57 years, and the female:male ratio was $1.9: 1$. In all cases, complete photo-documentation of the 21 gastric areas was achieved. The overall rate of detection of GIN was $2.8 \%$. Lowgrade displasia, high-grade dysplasia, and adenocarcinoma were found in 13 (2.3\%), 2 (0.3\%), and $1(0.2 \%)$ of the patients, respectively. The prevalence of at least 1 premalignant condition was $31 \%$, and helicobacter pylori infection was found in $57 \%$ of patients.

Conclusions: Using the SACE approach and with proper training, we have reported herein a high frequency of GIN in patients from a low socioeconomic status. Gastric cancer detection can be improved in a Western endoscopy setting when SACE, as a screening method, is performed by a trained endoscopist.

to $26 \%$ of GC were missed at an endoscopy performed up to 3 years before [5]. Because failure to detect subtle EGC at endoscopy may contribute to this poor prognosis, efforts should be made in the Western world to improve quality standards, with the aim of increasing chances for early diagnosis [5].

The Systematic Alphanumeric Coded Endoscopy (SACE) approach has been proposed to improve quality of EGD by facilitating complete examination of the upper gastrointestinal tract based on simple, sequential, and systematic overlapping photo-documentation, comprising 8 regions and 28 areas. In the stomach, the SACE protocol evaluates 5 regions and 21 areas, examining the entire gastric surface without any blind spots [6]. This novel method has proven its efficacy in Colombia, diagnosing $2(0,3 \%)$ EGCs in 650 male and female healthy volunteers of average risk [7]. Similar re- 


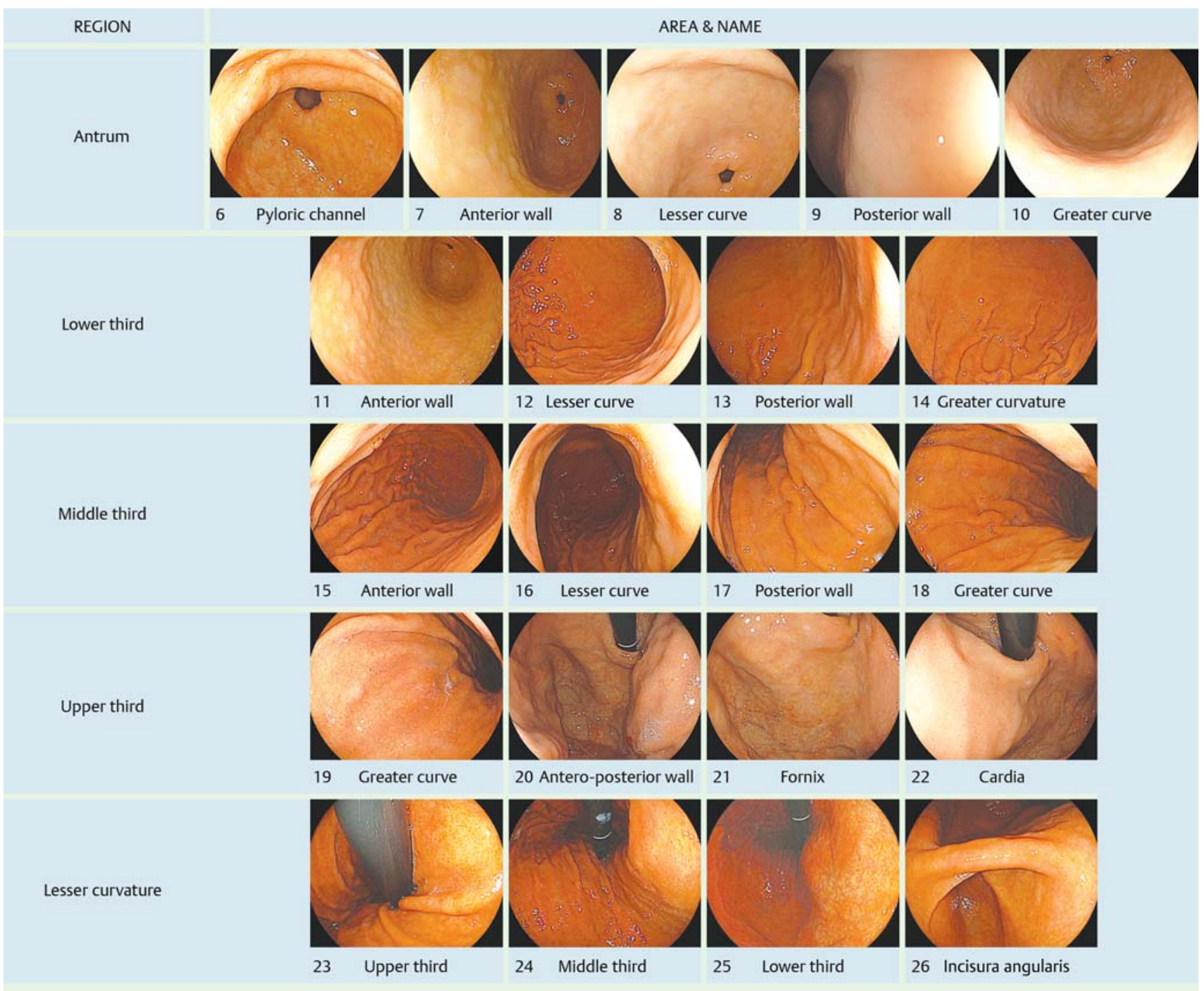

Fig. 1 Systematic alphanumeric approach of the stomach comprising 5 regions: antrum, lower third, middle third, upper third and lesser curvature, and 21 areas.

sults had been achieved in a cost-benefit endoscopic study in China, identifying $743(0.37 \%)$ GCs in a high-risk male cohort of 198,823 subjects [8]. Although the SACE procedure is a promising method of improving diagnosis of gastric intraepithelial neoplasia (GIN), studies on its effectiveness are scarce. We postulated that the SACE approach can increase the detection of GIN, and therefore, this prospective cross-sectional study was designed to determine the frequency in a -cohort of patients from a low socioeconomic level. Non-targeted biopsies also were used to evaluate the frequency of gastric premalignant conditions.

\section{Patients and methods}

$\nabla$

Patients from low socioeconomic level who qualified for the Peruvian Medicaid program, Sistema Integrado de Salud (SIS), aged 40 to 90 years, without previous EGD up to 3 years before the study was initiated, were consecutively enrolled between January 2013 and December 2014 at the Huacho Regional Hospital, in Huacho, Peru. SACE was indicated for both symptomatic patients and screening purposes. Patients with a history of gastric surgery, liver cirrhosis, those receiving nonsteroidal anti-inflam- matory drugs or proton pump inhibitors and those with signs of advanced digestive neoplasm were excluded. To minimize the variability in the endoscopy procedure (macroscopic assessment and biopsy sampling protocol), procedures were performed only by 1 trained gastroenterologist (N.M.). Information on clinicopathologic factors including age, gender, and family history of GC was obtained by the endoscopist who performed the procedure, who also obtained consent from the patient to be involved in the study. The Ethics Committee of the hospital approved the study protocol.

\section{Endoscopist's training background}

The Western endoscopist participating in this series (N.M.) is a graduate gastroenterologist with specific expertise in upper gastrointestinal endoscopy. Her additional training was at the EmuraCenter LatinoAmerica, Colombia, during an intensive 2-day hands-on training course focused on the SACE method and on early diagnosis [9]. Her training consisted of a step-by-step approach, encompassing a comprehensive review of the alphanumeric gastric codes and endoluminal anatomy, as well as training in diagnosis of EGC. Further hands-on training was obtained by practicing on stomach models, and then per- 
forming SACE in patients under the close supervision of highly experienced endoscopists. This course culminated with a handson practical examination and a written examination, after which university certification in SACE was awarded.

\section{Endoscopic evaluation}

With the patient in left lateral decubitus position, the gastric mucosa was washed out with $100 \mathrm{~mL}$ of Simethicone solution (Medifarma, Peru) $0.8 \mathrm{mg} / \mathrm{mL}$ to remove gastric foam and bubbles. The Simethicon solution was injected directly into the working channel of the endoscope with a 50-mL disposable syringe. The SACE method proposed by Emura et al, comprising 5 regions and 21 gastric areas, was routinely used for diagnosis [6] ( $\bullet$ Fig. 1). Examination of the stomach was initiated at the pyloric ring. Then, by using frontal view and while pulling backward, the antrum, the distal, and the middle third of the gastric corpus were examined, by rotating the endoscope in a clockwise manner. Once in the upper third greater curvature of the corpus, the gastric fornix, the cardia, the lesser curvature, and the incisura angularis, respectively, were examined by retroflexion view ( $\bullet$ Video 1 ). A total of 21 overlapping images corresponding to each gastric area were recorded in each patient. After each procedure, images were reviewed for completeness. When a suspicious lesion was found, Simethicone solution was applied to the lesion to wash out the overlying mucus and residue. Macroscopic types were determined based on the Paris classification [10], and the size of a lesion was estimated using the open width of a standard, fully opened $(7 \mathrm{~mm})$ hot biopsy forceps as a reference. Suspicious lesions were diagnosed using only white light, based on endoscopic signs suggestive of intraepithelial neoplasia such as uneven surface, paleness, redness color or spotty bleeding. The location was divided according to the SACE nomenclature ( $\bullet$ Table 1 ) [6]. The cross-sectional position on the circumference was divided into the anterior wall, lesser curvature, posterior wall, and greater curvature [11]. Gastroenterologist administered midazolam $3 \mathrm{mg}$ to $5 \mathrm{mg}$ when sedation was requested by the patient. Patients were monitored using continuous pulse oximetry and for blood pressure and heart rate.

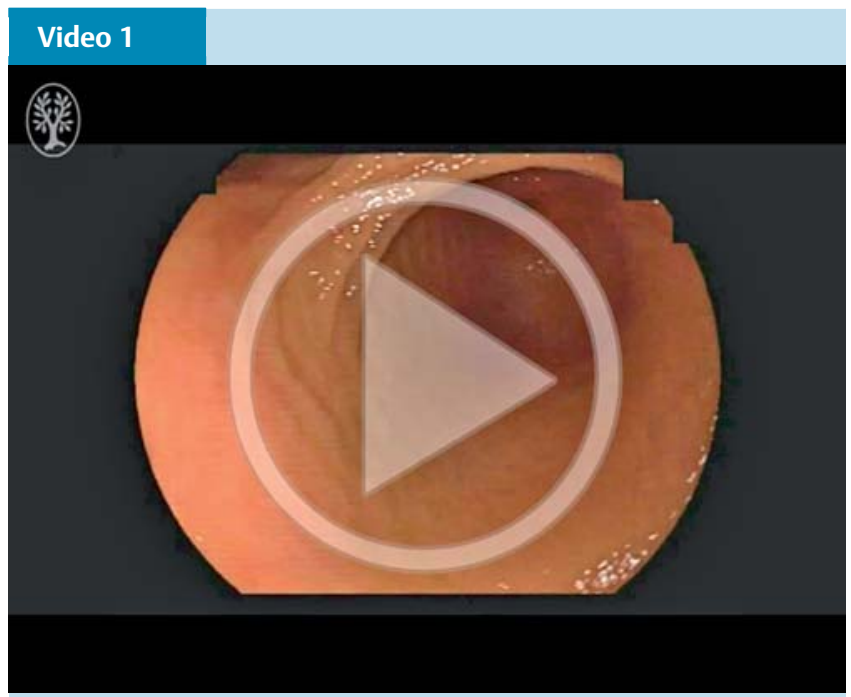

Gastric SACE procedure Online content including video sequences viewable at: http://dx.doi.org/10.1055/s-0042-115408
Table 1 SACE nomenclature of the upper digestive tract.

\begin{tabular}{|c|c|c|}
\hline Region & Area & Alphanumeric code \\
\hline Pharynx & Hypopharynx & P1 \\
\hline \multirow[t]{4}{*}{ Esophagus } & Upper third & E2 \\
\hline & Middle third & E3 \\
\hline & Lower third & E4 \\
\hline & Esophageal hiatus & E5 \\
\hline \multirow[t]{5}{*}{ Antrum } & Pyloric channel & A6 \\
\hline & Anterior wall & A7 \\
\hline & Lesser curvature & A8 \\
\hline & Posterior wall & A9 \\
\hline & Greater curvature & $\mathrm{A} 10$ \\
\hline \multirow{4}{*}{$\begin{array}{l}\text { Gastric body, } \\
\text { Lower third }\end{array}$} & Anterior wall & L11 \\
\hline & Lesser curvature & L12 \\
\hline & Posterior wall & L13 \\
\hline & Greater curvature & L14 \\
\hline \multirow{4}{*}{$\begin{array}{l}\text { Gastric body, } \\
\text { Middle third }\end{array}$} & Anterior wall & M15 \\
\hline & Lesser curvature & M16 \\
\hline & Posterior wall & M17 \\
\hline & Greater curvature & M18 \\
\hline \multirow{4}{*}{$\begin{array}{l}\text { Gastric body, } \\
\text { Upper third }\end{array}$} & Greater curvature & U19 \\
\hline & Antero-posterior wall & U20 \\
\hline & Fornix & U21 \\
\hline & Cardia & U22 \\
\hline \multirow[t]{4}{*}{ Lesser curvature } & Upper third & Lc23 \\
\hline & Middle third & Lc24 \\
\hline & Lower third & Lc25 \\
\hline & Incisura angularis & Lc26 \\
\hline \multirow[t]{2}{*}{ Duodenum } & Duodenal bulb & D27 \\
\hline & 2nd portion & D28 \\
\hline
\end{tabular}

\section{Endoscopic visibility}

Endoscopic visibility was graded from 1 to 3, according to Bhandari et al as follows: 1 , no adherent mucus and clear view of the mucosa; 2 , a thin coating of mucus but no obscured vision; and 3 , adherent mucus obscuring vision [12].

\section{Endoscopic equipment}

Procedures were performed by using a Fujinon EG ZW590 HD video endoscope, and a standard EPX-4450 video processor system (Fujifilm, Co, Japan). All images were saved in a compactflash XD card (SanDisk, Japan) and stored.

\section{Biopsy samples}

A targeted biopsy was obtained from any endoscopically detected focal lesion. In addition, 4 non-targeted biopsies of 2 topographic sites (at the lesser and greater curvature, from both the antrum and the corpus) were taken in accordance to the European MAPS guideline [13]. Biopsy samples were submitted to the pathology department in different vials labelled according to the site of the sample. Two expert gastrointestinal pathologists evaluated all specimens.

\section{Histopathologic analysis}

The specimens were immediately fixed in $10 \%$ formalin and embedded in paraffin. Serial cuts of $3 \mathrm{~mm}$ were stained with hematoxylin and eosin, and evaluated by two expert gastrointestinal pathologists. Helicobacter pylori (Hp) status was assessed by histology. Cases were considered Hp-positive when bacteria (irrespective of their density) were histologically detected in one or more of the available biopsy samples. Patients in whom Hp was not histologically detected at the time of the endoscopy proce- 
Table 2 Vienna Classification of intraepithelial neoplasia.

\begin{tabular}{|l|l|}
\hline Category 1 & Negative for neoplasia/dysplasia \\
\hline Category 2 & Indefinite for neoplasia/dysplasia \\
\hline Category 3 & Noninvasive low grade neoplasia/dysplasia \\
\hline Category 4 & Noninvasive high grade neoplasia \\
\hline & $\begin{array}{l}\text { 4.1 High-grade adenoma/dysplasia } \\
\text { 4.2 Noninvasive carcinoma (carcinoma in situ) }\end{array}$ \\
\hline Category 5 & Invasive neoplasia \\
\hline & 5.1 Intramucosal carcinoma \\
\hline
\end{tabular}

dure were considered Hp-negative. A premalignant lesion was defined as the presence of atrophy and or metaplasia in at least one biopsy sample, and these were assessed as present or absent according to the International Atrophy Group [14]. GIN was assessed according to the Vienna classification [15] ( $\bullet$ Table 2). ECG was defined as tumor invasion limited to the mucosa or submucosa, regardless of locoregional invasion [11].

\section{Management of lesions}

Patients with low-grade dysplasia (LGD) or high-grade dysplasia (HGD) without any visible lesion received eradication of $\mathrm{Hp}$, if present, and were re-examined 3 months after the procedure using FICE chromoendoscopy [16]. Visible LGD lesions were removed by endoscopic mucosal resection (EMR), whereas HGD, intramucosal and submucosal carcinoma, or beyond, were referred to surgical treatment. After EMR, FICE chromoendoscopy was used to evaluate the post-resection sites for any residual lesion.

\section{Statistical analysis}

Knowing that the reported prevalence of neoplastic lesions from the targeted population was as high as $1.9 \%$ [17], a sample size of 550 subjects was calculated (error margin: 1.5\%, CI: $99 \%$ ). Categorical variables were analyzed using the statistical package Stata 12.0 (Stata Corp LP). The Chi-Square Test was used to determine the association between categorical variables, where a $P$ value $<0.05$ was considered statistically significant.

\section{Results}

$\nabla$

A total of 601 subjects were enrolled in the study. Six cases were excluded due to incomplete SACE examination, and 22 cases due to incomplete biopsy report. Finally, 573 patients were analyzed. The mean age was 57 years, and the female:male ratio was 1.9:1.
In all cases, mucosal endoscopic visibility was graded as 1, no adherent mucus and clear view of the mucosa, and complete photodocumentation of the 21 gastric areas was achieved. There were no complications during or after the SACE examination, or due to biopsy sampling in any patient.

There were 3 ( $0.5 \%$ ) histologically confirmed cases of EGC, 2 diagnosed as HGD and 1 adenocarcinoma ( $\bullet$ Fig. 2). Macroscopic characteristics of ECGs are shown in Table 3. The overall detection rate for GIN was $2.8 \%(16 / 573)$. LGD, HGD, and adenocarcinoma were found in $13(2.3 \%), 2(0.3 \%)$ and $1(0.2 \%)$ patients, respectively. A total of 17 GINs were diagnosed in 16 patients, with 1 patient having 2 lesions with LGD. All protruding lesions (Is, Isp) were diagnosed as LGD, meanwhile, 3 (60\%) out of 5 flat superficial lesions (IIa, IIa + IIc, IIc) were diagnosed as EGCs ( $\bullet$ Table 4$)$. Mean size of EGCs and LGDs was $11.3 \mathrm{~mm}$ and $10 \mathrm{~mm}$, respectively. Lesions were located in the pyloric channel, antrum, incisura angularis, lower third, middle third, and upper third in $23.5 \%$, $23.5 \%, 0 \%, 23.5 \%, 11.8 \%, 5.9 \%$ and $11.8 \%$ of the cases, respectively. Regarding cross-sectional position on the circumference, most of the 11 lesions, exclusive of the 6 lesions involving the pyloric channel and the cardia, were located in the anterior wall $(36.4 \%, 4 / 11)$ followed by the lesser curvature $(27.3 \%, 3 / 11)$, and the posterior $(18.2 \%, 2 / 11)$ and the greater curvature $(18.2 \%, 2 / 11)(-$ Table 5$)$. Post-EMR histopathology revealed LGD and free horizontal margin in all resected specimens ( Table 6 ). There were 5 cases of non-visible lesions diagnosed as LGD, which were derived from the biopsy protocol. Four of these cases were Hp-positive and received eradication treatment. Follow-up endoscopy including targeted biopsy using FICE, reported metaplasia without dysplasia in all cases. There were no cases of non-visible HGD. On the other hand, of a total of 54 suspicious lesions, 37 (68\%) were diagnosed as non-neoplastic (gastritis, metaplasia, hyperplastic polyp, inflammatory polyp) by histopathology.

As for premalignant conditions, atrophy was found in $3(0.5 \%)$, atrophy and metaplasia in $6(1 \%)$, and intestinal metaplasia without atrophy in 171 (30\%) of the patients. The overall prevalence of at least 1 premalignant condition was $31 \%$. There was no association between clinicopathologic factors and patients diagnosed as having no premalignant, premalignant, and neoplastic lesions. Frequency of premalignant conditions, was higher in patients older than age $60(P<.02)$. Hp positivity was found in $206 / 384$ (53.6\%), 114/173 (66\%), and 6/16 (37.5\%) of patients diagnosed as having no premalignant, premalignant, and neoplastic lesions, respectively. Overall, the $\mathrm{Hp}$-positive rate was $57 \%$. Intravenous sedation with midazolam was administered to 469 (82\%) of patients. No differences were found in the detection of intraepithelial neoplasia in patients receiving or not receiving sedation.
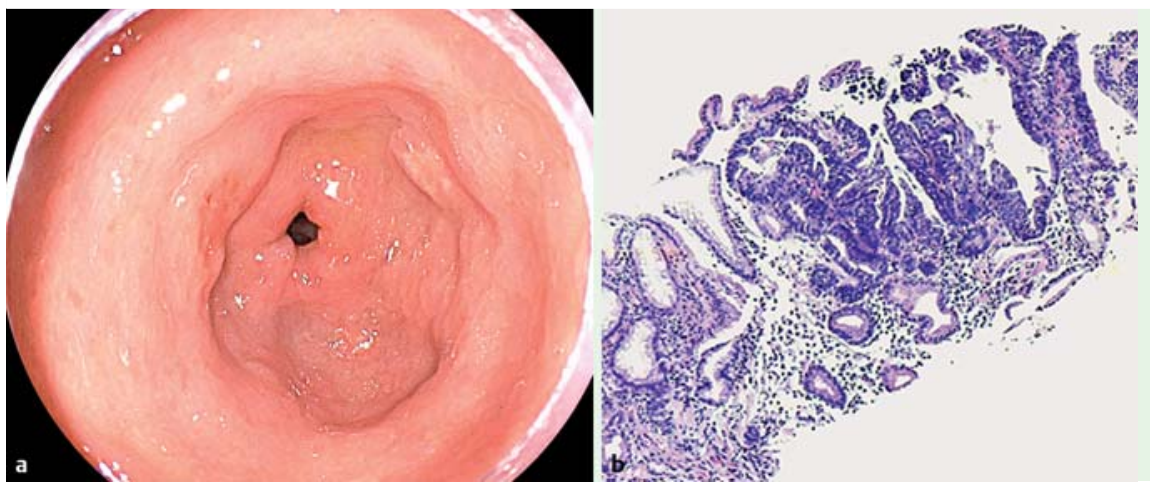

Fig. 2 a lla +llc lesion $7 \mathrm{~mm}$ in diameter located on the antrum anterior wall. $\mathbf{b}$ Histopathology revealed high-grade dysplasia with typical distortion of the foveolar architecture, cell proliferation, and increased nucleus/cytoplasm relationship. 
Table 3 Macroscopic characteristics of EGCs.

\begin{tabular}{|llll|}
$\begin{array}{l}\text { Macroscopic } \\
\text { type }\end{array}$ & Color & $\begin{array}{l}\text { Spotty } \\
\text { bleeding }\end{array}$ & Histopathology \\
\hline Ila & Redness & Postive & HGD \\
\hline IIC & Redness & Negative & HGD \\
\hline Ila + IIC & Redness & Positve & Intramucosal adenocarcinoma \\
\hline
\end{tabular}

HGD, high-grade dysplaisa.

Table 4 Macroscopic types and histopathology.

\begin{tabular}{|lll|}
$\begin{array}{l}\text { Macroscopic } \\
\text { types }\end{array}$ & LGD & $\begin{array}{l}\text { HGD/intramucosal } \\
\text { cancer }\end{array}$ \\
\hline Is/Isp & $12(100 \%)$ & 0 \\
\hline Ila, Ila + IIc, IIc & $2(30 \%)$ & $3(60 \%)$ \\
\hline
\end{tabular}

LGD, low-grade dysplasia; HGD, high-grade dysplasia.

Table 5 Location of GIN and histopathology.

\begin{tabular}{|llll|}
\hline Location & Number/percentage & LGD & HGD \\
\hline Pyloric channel & $4(23.5)$ & 3 & 1 \\
\hline Antrum & $4(23.5)$ & 3 & 1 \\
\hline Incisura angularis & 0 & - & - \\
\hline Lower third & $4(23.5)$ & 3 & 1 \\
\hline Middle third & $2(11.8)$ & 2 & 0 \\
\hline Upper third & $1(5.9)$ & 1 & 0 \\
\hline Cardia & $2(11.8)$ & 2 & 0 \\
\hline & & & \\
\hline Anterior wall & $4(36.4)$ & 3 & 1 \\
\hline Lesser curvature & $3(27.3)$ & 2 & 1 \\
\hline Posterior wall & $2(18.2)$ & 2 & 0 \\
\hline Greater curve & $2(18.2)$ & 2 & 0 \\
\hline
\end{tabular}

LGD, low-grade dysplasia; HGD, high-grade dysplasia.

Table 6 Histopathologic results of LGD post-EMR.

\begin{tabular}{|lll|}
\hline Paris type & Size $(\mathbf{m m})$ & Post-EMR histopathology \\
\hline Is & 15 & LGD \\
\hline Is & 10 & LGD \\
\hline Isp & 10 & LGD \\
\hline Is & 9 & LGD \\
\hline Isp & 10 & LGD \\
\hline Is & 7 & LGD \\
\hline Is & 4 & LGD \\
\hline Ila + Ilc & 9 & LGD \\
\hline Is & 7 & LGD \\
\hline Isp & 10 & LGD \\
\hline Is & 11 & LGD \\
\hline Is & 12 & LGD \\
\hline Illa & 10 & LGD \\
\hline Is & 15 & LGD \\
\hline
\end{tabular}

LGD, low-grade displasia; EMR, endoscopic mucosal resection.

\section{Discussion}

$\nabla$

Despite extensive worldwide use of EGD examinations, GC is usually diagnosed at an advanced stage in the western world. Although several recommendations have been promulgated [18, 19], currently performed standard EGD is not a standardized procedure as it lacks sequentiality, order, proper nomenclature, and a protocol for completeness. These limitations could be partially responsible for the high estimated rate of missed lesions during
EGD [5], and the perceived scarcity of EGC in western countries with high prevalence of the disease [9].

By using the SACE approach, we have reported herein $3(0.5 \%)$ EGCs in 573 asymptomatic or dyspeptic patients. This result shows a higher frequency of GC than that reported in other western screening studies $[8,20]$. A previous study using SACE as the screening method reported 2 (0.3\%) ECGs in 650 volunteers in Colombia [8], and another using standard EGD reported 45 (0.14\%) GCs in 32,388 Peruvian subjects, with no alarm symptoms [20]. On the other hand, earlier Asian screening studies using standard EGD reported $81(0.4 \%)$ GCs in 18,414 volunteers in Korea [21], and 41 (1.9\%) GCs in 2,192 volunteers in Japan [17]. Although it is known that Huacho Hospital serves a rural low socioeconomic population, where up to $76.7 \%$ of the inhabitants have at least 1 basic unmet need, studies on the prevalence of GIN were lacking. In fact, these 3 EGCs constitute the first cases diagnosed at the Huacho Hospital and by the physician participating in this study. Although ESD has become an excellent alternative for the treatment of ECG, the lack of both training and appropriate ESD devices currently preclude us from performing this procedure, and therefore, patients were referred for surgical treatment.

There were fourteen (2.4\%) visible lesions diagnosed as LGD. This result constitutes one of the highest frequencies of gastric LGD reported in a western screening study, as previous studies have reported rates varying from $0.5 \%$ to $3.75 \%[22,23]$. Higher frequencies of LGD varying from $9 \%$ to $20 \%$ have been reported in Asian countries, where the prevalence of GC is high [24]. All these lesions were resected by EMR for full histologic evaluation and for the risk of HGD or carcinoma in other parts of the lesion [13, 24]. The overall rate of detection of GIN in this study (2.8\%) is 14 times higher than that in an earlier Peruvian study using standard EGD, which reported gastric neoplasia in $0.2 \%$ of patients [25].

There are several explanations for these results. SACE, as a sequential, alphanumeric, overlapped no-blind-spots examination, is part of an endoscopic invention presented to the US Patent Office in March 2011 by Emura et al. [26]. It facilitates sequential examination of the entire gastric surface by a consecutive registry of 21 areas, with 21 overlapped images. Noteworthy, registered images allow cross-examination, potentially reducing the bias of the performing endoscopist. The concept of a consecutive image registry affords examiners the order in which the exam should be performed, and the overlapped images are fundamental to considering SACE as a no-blind-spots examination. Another advantage is that for precise localization of normal or abnormal areas, the method encompasses a novel endoluminal alphanumeric nomenclature, based on the identification of natural axes, walls, curvatures, and anatomical landmarks [6]. On the other hand, gastric SACE is in practice as part of a complete systematic evaluation of the upper gastrointestinal tract, which includes the pharynx, the esophagus, and the duodenum. Considering these features, SACE differs from a previous 4-image study [19] and from a recent proposal for 22 non-overlapped, nonnumerical images [27].

Gastric premedication before the procedures was determinant to obtain clear mucosal visualization in all cases. As reported, simethicone without a mucolytic agent has provided clear visibility of the mucosa, reducing the amount of gastric foam and bubbles [24]. Although a Simethicon solution of $100 \mathrm{~mL}$ before the procedure has been recommended [29], we used intragastric lavage to facilitate target cleaning of focal areas and also to rinse suspicious lesions. 
Because it has been suggested that less experienced endoscopists have a higher rate of missing lesions during endoscopy [5], the step-by-step training in detection and characterization of neoplastic lesions received by the participant endoscopist also contributed, in part, to the results of this study. Even though improved endoscopic visibility with use of simethicone and training of the participant endoscopist played an important role, we believe that SACE as the screening method was the main factor that contributed to achieving these results. Further research validating this approach, and randomized studies with and without premedication are, however, necessary to confirm our findings. There were 5 cases of non-visible lesions diagnosed as LGD. As reported, non-visible LGD may appear as a minute isolated or multifocal lesion, difficult to detect during a second examination [13]. Although of limited availability in Peru, HD scopes coupled to FICE-enhanced endoscopy were used to reexamine these patients, finding no neoplasia 3 months after the procedure. Because this assumed disappearance does not rule out the possible progression to cancer, these patients are currently in an endoscopic follow-up program.

Our study demonstrated at least 1 premalignant condition in $31 \%$ of the patients, and these lesions were statistically more frequent in those older than age 60.These results are comparable to $30 \%$ rate of gastric premalignant conditions reported in Colombia, in a cohort of patients from low socioeconomic level and similar ages, that also reported a high frequency of premalignant lesions in elders [7]. On the other hand, 2 Peruvian studies in subjects of middle and high socioeconomic status have reported rates of premalignant lesions as low as $7.1 \%$ to $8.2 \%[25,30]$. Taken together, these differences can be explained based on the known relationship between low socioeconomic level and GC [4], and warrant the implementation of GC screening programs targeting this high-risk subpopulation.

The number of biopsies regarding staging of gastric premalignant lesions is quite controversial, mainly because of the multifocal nature of these lesions [31,32]. To determine this frequency, we sampled 4 sites as recommend by Satoh et al. [32] and based on the recent European guidelines for atrophy evaluation [13]. Categorizing the risk of progression to GC that requires 5 biopsies was not considered in this study. Hp infection was found in $57 \%$ of the patients. Identical results were reported in another Peruvian study in asymptomatic subjects [30], but not in a Colombian study in which Hp infection was reported in $75 \%$ of participants [7]. Based on the reported strong association between Hp infection and GC [13], a higher rate of Hp-infected cases would be expected in this study's population. Gastric carcinogenesis is, however, a process that cannot be attributed to Hp infection exclusively, but rather to complex individual, environment, dietary, and socioeconomic interactions [2].

Compared to Asian mass screening studies, a limitation of this study was the relatively low number of screened patients. In addition, the length of the procedure was not herein evaluated. We are currently performing another study quantifying the time frame of the SACE approach compared to standard EGD. Another limitation is that the outcomes represent the experience of an individual trained endoscopist, and may not necessarily be extrapolatable to other western endoscopy settings. In addition, although it seems use of SACE has expanded in practice [33], further validation studies are needed to establish it as a frontline method of mass screening.

Because EGDs performed in western countries are less accurate than those performed in Asia [34] and the diagnostic accuracy of
HD endoscopy for GIN is significantly higher than that of standard-definition endoscopy [35], future adoption of SACE implemented with widespread use of HD coupled to IEE would significantly increase GIN detection rates worldwide.

\section{Conclusion \\ $\nabla$}

In summary, by using the SACE approach and with proper training, we were able to diagnose a high frequency of GIN in a cohort of patients from low socioeconomic levels.

Given the impact on patients and their relatives of an endoscopic procedure that often fails to diagnose cancer, as well as the costs of more invasive treatment and the reduction in quality of life for patients, SACE constitutes a promising alternative to improve the quality of EGD in the western world, and can be adopted in the future as the preferred method for gastric screening examination.

\section{Competing interests: None}

\section{Institutions}

1 Department of Medicine, Hospital Regional de Huacho, Lima, Peru

2 Advanced Gastrointestinal Endoscopy, EmuraCenter LatinoAmerica, Bogotá, Colombia

${ }^{3}$ Emura Foundation for the Promotion of Cancer Research, Bogotá, Colombia

${ }^{4}$ Division of Gastroenterology, Universidad de La Sabana, Bogotá, Colombia

${ }^{5}$ Service of Gastroenterology, Department of Medical Specialties, National

Institute of Neoplastic Diseases, Lima, Peru

${ }^{6}$ Department of Pathology, Hospital Nacional Daniel Alcides Carrión, Lima, Peru

${ }^{7}$ Hospital Guillermo Kaelin de la Fuente, Essalud, Lima, Peru

${ }^{8}$ School of Medicine, Faculty of Health Sciences, Peruvian University of Applied Sciences, Lima, Peru

\section{Acknowledgements}

$\nabla$

The authors would like to thank Anne Shiwa from Global Editing \& Translation Services for editing this manuscript. This work was supported in part by a grant-in-aid for the Comprehensive Strategy to Control Cancer in the Americas from the Emura Foundation for the Promotion of Cancer Research.

\section{References}

1 Ferlay J, Soerjomataram I, Ervik M et al. Globocan 2012 v. 1.0. Cancer Incidence and Mortality Worldwide: IARC CancerBase No. 11. 2013

2 Crew KD, Neugut AI. Epidemiology of gastric cancer. World J Gastroenterol 2006; 12: 354-362

3 Mendoza D, Herrera P, Gilman RH et al. Variation in the prevalence of gastric cancer in Peru. Int J Cancer 2008; 123: 414-420

4 Roder DM. The epidemiology of gastric cancer. Gastric Cancer 2002; 5: (Suppl. 01): 5-11

5 Menon S, Trudgill N. How commonly is upper gastrointestinal cancer missed at endoscopy? A meta-analysis. Endosc Int Open 2014; 2: E46-50

6 Emura F, Gralnek I, Baron TH. Improving early detection of gastric cancer: a novel systematic alphanumeric-coded endoscopic approach. Rev Gastroenterol Peru 2013; 33: 52 -58

7 Emura F, Mejía J, Mejía M et al. Effectiveness of systematic chromoendoscopy for diagnosis of early cancer and gastric premalignant lesions. Results of two consecutive screening campaigns in Colombia (20062007). Rev Col Gastroenterol 2010; 25: 19-30

8 Dan YY, So JB, Yeoh KG. Endoscopic screening for gastric cancer. Clin Gastroenterol Hepatol 2006; 4: 709-716

9 Emura F, Mejía J, Donneys A et al. Therapeutic outcomes of endoscopic submucosal dissection of differentiated early gastric cancer in a Western endoscopy setting (with video). Gastrointest Endosc 2015; 82: $804-811$ 
10 Endoscopic Classification Review G. Update on the paris classification of superficial neoplastic lesions in the digestive tract. Endoscopy 2005; 37: $570-578$

11 Japanese Gastric Cancer Association. Japanese gastric cancer treatment guidelines 2010 (ver. 3). Gastric Cancer 2011; 14: 113-123

12 Bhandari P, Green S, Hamanaka $H$ et al. Use of gascon and pronase either as a pre-endoscopic drink or as targeted endoscopic flushes to improve visibility during gastroscopy: a prospective, randomized, controlled, blinded trial. Scand J Gastroenterol 2010; 45: 357-361

13 Dinis-Ribeiro $M$, Areia $M$, de Vries AC et al. Management of precancerous conditions and lesions in the stomach (MAPS): guideline from the European Society of Gastrointestinal Endoscopy (ESGE), European Helicobacter Study Group (EHSG), European Society of Pathology (ESP), and the Sociedade Portuguesa de Endoscopia Digestiva (SPED). Endoscopy 2012; 44: 74-94

14 Rugge M, Correa P, Dixon MF et al. Gastric mucosal atrophy: interobserver consistency using new criteria for classification and grading. Aliment Pharmacol 2002; 16: 1249-1259

15 Schlemper RJ, Riddell RH, Kato $Y$ et al. The Vienna classification of gastrointestinal epithelial neoplasia. Gut 2000; 47: 251-255

16 Jung SW, Lim KS, Lim JU et al. Flexible spectral imaging color enhancement (FICE) is useful to discriminate among non-neoplastic lesion, adenoma, and cancer of stomach. Dig Dis Sci 2011; 56: 2879-2886

17 Hosokawa O, Miyanaga T, Kaizaki Y et al. Decreased death from gastric cancer by endoscopic screening: association with a population-based cancer registry. Scand J Gastroenterol 2008; 43: 1112-1115

18 American Society for Gastrointestinal Endoscopy. The role of endoscopy in the surveillance of premalignant conditions of the upper gastrointestinal tract. Gastrointest Endosc 1998; 48: 663 - 668

19 Rey JF, Lambert R. ESGE Quality Assurance Committee. ESGE recommendations for quality control in gastrointestinal endoscopy: guidelines for image documentation in upper and lower GI endoscopy. Endoscopy 2001; 33: $901-903$

20 Uehara G, Nago A, Espinoza $R$ et al. Optimal age for gastric cancer screening in patients with dyspepsia without alarm symptoms. Rev Gastroenterol Peru 2007; 27: 339-348

21 Nam SY, Choi IJ, Park KW et al. Effect of repeated endoscopic screening on the incidence and treatment of gastric cancer in health screenees. Eur J Gastroenterol Hepatol 2009; 21: 855-860

22 Farinati $F$, Rugge $M$, Di Mario $F$ et al. Early and advanced gastric cancer in the follow-up of moderate and severe gastric dysplasia patients. A prospective study. I.G.G.E.D. - Interdisciplinary Group on Gastric Epithelial Dysplasia. Endoscopy 1993; 25: $261-264$

23 Bearzi I, Brancorsini D, Santinelli A et al. Gastric dysplasia: a ten-year follow-up study. Pathol Res Pract 1994; 190: 61 -68

$24 \mathrm{Kim}$ J-W, Jang JY. Optimal management of biopsy-proven low-grade gastric dysplasia. World J Gastrointest Endosc 2015; 7: 396-402

25 Chacaltana A, Rodriguez C, Urday C et al. Lesiones gastricas preneoplasicas y helicobacter pylori en despistaje endoscopico para cáncer gástrico en población de nivel socioeconomico medio y alto. Rev Gastroenterol Peru 2009; 29: 218-225

26 Systematic chromoendoscopy and chromocolonoscopy as a novel systematic method to examine organs with endoscopic techniques. Available at: http://www.google.ch/patents/US8579800. Accessed April 26, 2016

27 Veitch AM, Uedo N, Yao K et al. Optimizing early upper gastrointestinal cancer detection at endoscopy. Nat Rev Gastroenterol Hepatol 2015; 12: $660-667$

28 Ahsan M, Babaei L, Gholamrezaei A et al. Simethicone for the Preparation before Esophagogastroduodenoscopy. Diagn Ther Endosc 2011; 2011: 484532

29 Chang WK, Yeh MK, Hsu HC et al. Efficacy of simethicone and N-acetylcysteine as premedication in improving visibility during upper endoscopy. J Gastroenterol Hepatol 2014; 29: 769 - 774

30 Ramirez Ramos A, Recavarren Arce S, Arias Stella J et al. Helicobacter Pylori, Chronic Gastritis, Gastric And duodenal Ulcer: Study of 1638 Patients. Rev Gastroenterol Peru 1999; 19: 196-201

31 Guarner J, Herrera-Goepfert R, Mohar A et al. Diagnostic yield of gastric biopsy specimens when screening for preneoplastic lesions. Hum Pathol 2003; 34: $28-31$

32 Satoh K, Kimura K, Taniguchi Y et al. Biopsy sites suitable for the diagnosis of Helicobacter pylori infection and the assessment of the extent of atrophic gastritis. Am J Gastroenterol 1998; 93: 569-73

33 Rollan A, Cortes P, Calvo A et al. Recommendations of the Chilean Association for Digestive Endoscopy for the management of gastric pre-malignant lesions. Revista medica de Chile 2014; 142: 1181 1192

34 Hassan C, Zullo A, Cristofari F et al. Upper endoscopy in Europe: are we missing gastric cancer? J Clin Gastroenterol 2010; 44: 73

35 Toyoizumi $H$, Kaise $M$, Arakawa $H$ et al. Ultrathin endoscopy versus high-resolution endoscopy for diagnosing superficial gastric neoplasia. Gastrointest Endosc 2009; 70: 240-245 